\title{
3D in-situ imaging of cracks in concrete using diffuse ultrasound
}

Journal Title

$\mathrm{XX}(\mathrm{X}): 1-11$

(C)The Author(s) 2016

Reprints and permission:

sagepub.co.uk/journalsPermissions.nav

DOI: $10.1177 /$ ToBeAssigned

www.sagepub.com/

@SAGE

\section{Yuxiang ZHANG ${ }^{1}$, Eric Larose ${ }^{1}$, Ludovic Moreau ${ }^{2}$, and Grégoire d'Ozouville $^{3}$}

\begin{abstract}
Locadiff, an innovative imaging technique based on diffuse waves, has recently been developed in order to image mechanical changes in heterogeneous geological or man-made materials. This manuscript reports the on-site application of Locadiff to locate several pre-existing cracks on an aeronautical wind tunnel made of pre-stressed concrete. Using 32 transducers working at ultrasonic frequencies $(80-220 \mathrm{kHz})$ where multiple scattering occurs, we monitor during 15 minutes an area of $2.5 \mathrm{~m} \times 2.5 \mathrm{~m}$ of a $35 \mathrm{~cm}$ thick wall. With the wind tunnel in its routine operation, structural changes around the cracks are detected thanks to their closing or opening due to slight pressure changes. By mapping the density of such microstructure changes in the bulk of the material, the locating of three pre-existing cracks is properly performed in 3D.
\end{abstract}

\section{Keywords}

3D imaging, crack in concrete, in-situ, diffuse ultrasound, wind tunnel

${ }^{1}$ ISTerre, CNRS, BP53, 38041 Grenoble Cedex 9, France

${ }^{2}$ ISTerre, Université Grenoble-Alpes, France

${ }^{3}$ ONERA, Site du Fauga-Mauzac, 31410 Mauzac, France

Corresponding author:

Eric Larose

Email: eric.larose@univ-grenoble-alpes.fr

Prepared using sagej.cls [Version: 2015/06/09 v1.01] 


\section{Introduction}

Ultrasonic (US) waves constitutes an important family of non-destructive testing and evaluation (NDT\&E) methods, the applications of which have proved to be effective and efficient in various fields (e.g. medicine, aeronautics, automotive, etc.). However, when applied to heterogeneous materials such as concrete, the performance of conventional US methods (e.g. pulse-echo, impact-echo, tomography) is limited due to intrinsic and scattering attenuation of ballistic and coherent waves. Probed by ultrasonic waves, concrete is a multiple scattering medium in which the acoustic energy of direct waves rapidly converts into late arriving diffuse waves. The subsequent loss of amplitude of the coherent waves often results in a significant reduction of penetration depth and signal quality.

To reduce these inconveniences, the approach commonly adopted by conventional US methods is to lower the frequency, which inevitably impairs the sensitivity of the technique to detect the anomalies in the bulk of the material ${ }^{1}$. In addition, the directivity of US waves, which is critical for conventional US imaging method (e.g. B-scan, C-scan), is also degraded.

For these reasons, efforts have been made on developing NDT\&E methods based on the use of diffuse US waves. Coda Wave Interferometry (CWI), originally developed for seismogram analysis ${ }^{2}$ in geosciences, has also been proposed for laboratory experiments on rocks ${ }^{3}$ and concrete samples ${ }^{4}$. Coda (multiply scattered) waves are known for being highly repeatable and sensitive to perturbations of the propagation medium $^{5}$. Thanks to this sensitivity, CWI allows the monitoring of the mechanical properties of concrete with a high precision ${ }^{6 ; 7}$. In view of applying CWI to detect the formation of damage in concrete, we can identify at least two approaches: the first one is based on monitoring the acousto-elasticity of concrete from apparent relative velocity changes ${ }^{8-11}$, while the second one is based on monitoring the change in its microstructure through the decorrelation of Coda waves ${ }^{12-17}$. To author's knowledge, the application of CWI and Locadiff for detecting several pre-existing cracks in-situ has not yet been reported, though the in-situ detection and cartography of existing cracks is a major concern of the NDT\&E community and in the industry. To investigate the capacity of the method to image pre-existing cracks, we propose to apply Locadiff on an aeronautical wind tunnel made of reinforced and pre-stressed concrete in an industrial context.

Prepared using sagej.cls 


\section{Method}

In order to study aircraft high-lift configurations at high Reynolds numbers, ONERA (the French national aerospace research establishment) built the F1 pressurized, subsonic wind tunnel in 1974, at its Fauga-Mauzac Test Centre near Toulouse - France. The pressure inside the tunnel cycles from atmospheric pressure to 3.85 bars, at least once a day, nearly every working day of the year. F1 is a closed-return circuit wind tunnel (72 m long and $18 \mathrm{~m}$ wide) with an overall length of $180 \mathrm{~m}$ (Fig. 1a). The biggest internal diameter of the circuit is $12 \mathrm{~m}$, and the depth of concrete is about $35 \mathrm{~cm}$. Most of this circuit consists of a sealed pressure shell built of pre-stressed reinforced concrete. Since the beginning of the wind tunnel operation, cracks are visible inside and outside the concrete pressure shell. When the circuit is pressurized, most cracks have air blowing through them. A total leakage rate is estimated around $3.5 \mathrm{~kg} / \mathrm{s}$ when the structure is in operation.
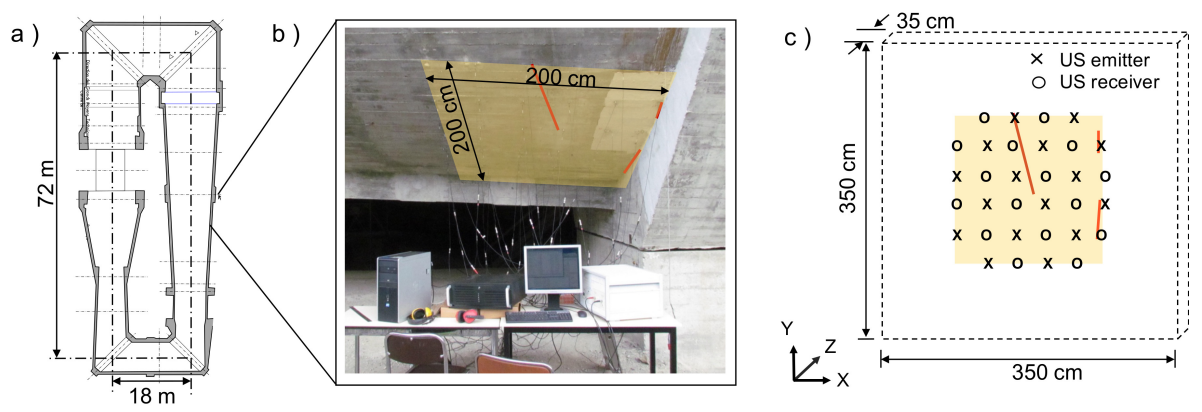

Figure 1. Experimental setup: a) schematic of the horizontal cut of the wind tunnel, the diameter of which ranges from 6 to $12 \mathrm{~m}$; b) photography of the experimental setup for the on-site measurements. 32 piezoelectric transducers are glued onto the outer surface of the concrete tunnel, homogeneously distributed within the yellow area; c) because the wall thickness is much smaller than the diameter of the tunnel, we approximate this part of the shell as a plane wall. Moreover, because of the strong attenuation of ultrasound and the size of the structure, we model the medium as infinite in the $X$ and $Y$ directions. The part of the shell at test is therefore modeled as an infinite concrete slab of thickness $35 \mathrm{~cm}$ with transducers in the yellow area.

Covering a small region of the tunnel (Fig. 1a) where three cracks are visually detected, 32 identical piezoelectric transducers are attached on the outer surface of the tunnel for US acquisition (Fig. 1b). Acting as 16 emitters and 16 receivers, they are evenly spaced $40 \mathrm{~cm}$ apart over a $2 \mathrm{~m} \times 2 \mathrm{~m}$ area. A chirp signal with frequency increasing from $80 \mathrm{kHz}$ to $220 \mathrm{kHz}$ is generated, amplified and sent into concrete through one of the emitters. Synchronized to each emission, receivers collect the waveforms all together at a $2.5-\mathrm{MHz}$ sampling frequency. To improve signal quality, 
this procedure is repeated 200 times before switching to the next emitter, and the average of the 200 waveforms measured at a given receiver is correlated with the source waveform to estimate the impulse response. The resulting signal is then stored as one coda signal for each transmit-receive pairs. The complete set of measurements consists of 256 coda signals ( 16 emitters $\times 16$ receivers) recorded within 3 minutes.

In Fig. 2a, an example of recorded coda signal is plotted together with its envelope, the square-root of its intensity. By fitting theoretical predictions of the intensity from the diffusion equation to those obtained experimentally from coda signals (Fig. 2b), the transport parameters of concrete, namely the diffusivity $\mathcal{D}$ and dissipation parameter $\xi$, are evaluated to be $\mathcal{D}=70 \mathrm{~m}^{2} / \mathrm{s}$ and $\xi=5500 \mathrm{~s}^{-1}$. With the propagation velocity estimated to be $c_{0} \approx 2500 \mathrm{~m} / \mathrm{s}$, the transport mean free path $\left(\ell^{\star}=3 \mathcal{D} / c_{0}\right)$ is of the order of $8 \mathrm{~cm}$, corresponding to a propagation time of $t^{\star}=0.03 \mathrm{~ms}$. Compared to $\ell^{\star}$, the distances between transducers is long enough $(\geq 40 \mathrm{~cm})$ to guarantee that recorded US waves are multiply scattered.

Within a 10 minutes interval, two sets of measurements are taken at the end of a pressurization cycle. During this period of time, the wind tunnel is slowly depressurizing, and the pressure inside slightly drops from $128.8 \mathrm{kPa}$ to $128.1 \mathrm{kPa}$. CWI is applied on the experimental coda using the stretching method ${ }^{18 ; 19}$ in order to evaluate the relative velocity change and the remnant decorrelation coefficients (DC). From multiple time-windows located between $0.4 \mathrm{~ms}$ and $1.9 \mathrm{~ms}$ in the coda, 1200 decorrelation values were calculated and retained as reliable observations with good signal-to-noise ratio (SNR). Compared to that reported in the literature ${ }^{12}$, observed decorations in this study are relatively small: the average value is $0.21 \%$ and $90 \%$ of them are less than $0.05 \%$.

A linear model ${ }^{13}$ is used to relate the DC values to the local microstructure changes characterized by the density of change in effective scattering cross-section, $\sigma(x)$, such that:

$$
\mathrm{DC}=\int_{\Omega} \frac{c_{0}}{2} K(x) \sigma(x) d x .
$$

In Eq.1, the total decorrelation is expressed as the integral of $\sigma$ over the propagation medium, $\Omega$, weighted by a term composed of the propagation velocity $c_{0}$ and the sensitivity kernel of corresponding diffuse waves $K(x)$. For diffuse waves emitted at $\mathbf{s}$ and detected at $\mathbf{r}$ after a period of time $t, K(\mathbf{s}, \mathbf{r}, t, x)$ estimates the density of time spent at $x$ from a statistical point of view:

$$
K(\mathbf{s}, \mathbf{r}, t, x)=\frac{\int_{0}^{t} I(\mathbf{s}, x, u) I(x, \mathbf{r}, t-u) d u}{I(\mathbf{s}, \mathbf{r}, t)}
$$

Prepared using sagej.cls 

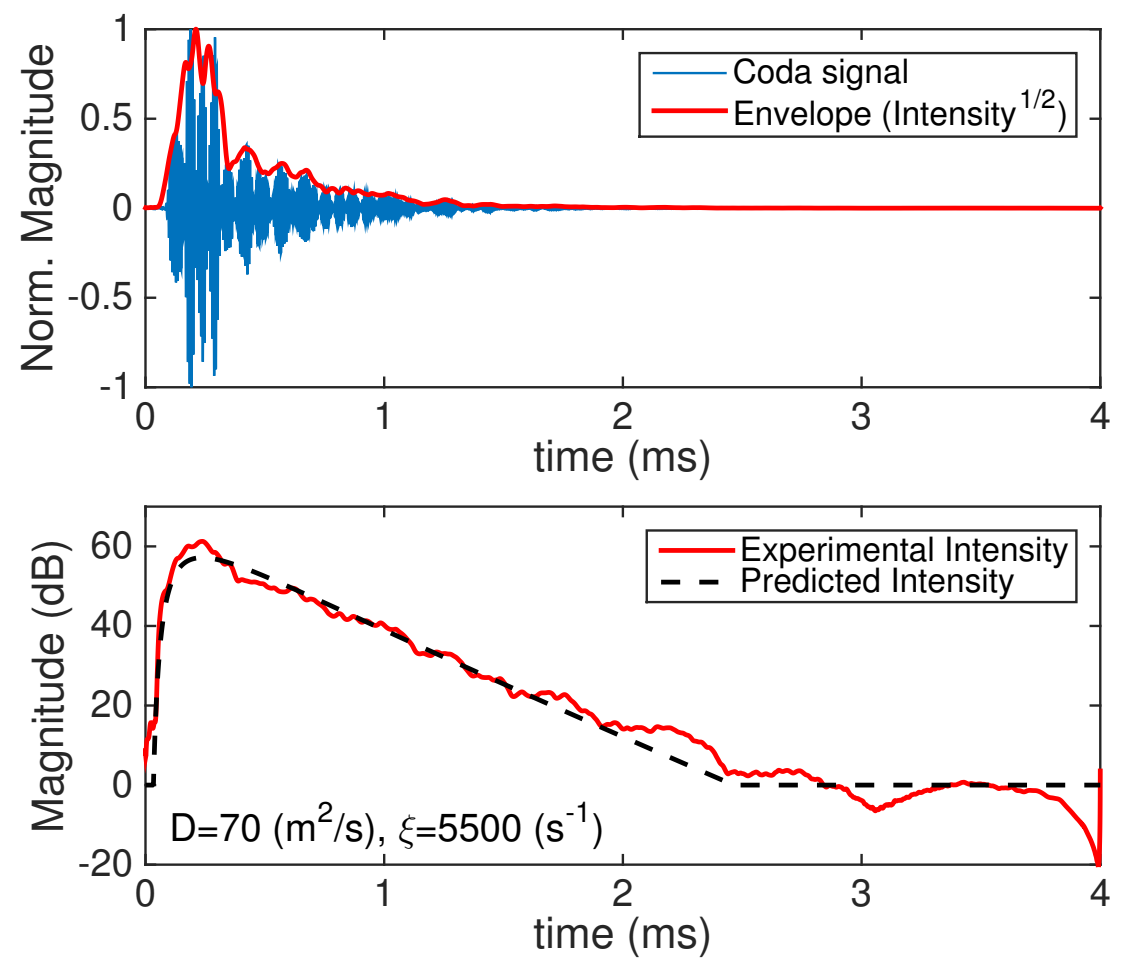

Figure 2. Illustration of a coda signal recorded using transducers that are $40 \mathrm{~cm}$ apart. Top: coda signal and its envelope (the square root of its intensity). Bottom: the comparison between the experimental intensity and the theoretical one using the diffusion equation with parameters $D=70\left(\mathrm{~m}^{2} / \mathrm{s}\right)$ and $\xi=5500\left(\mathrm{~s}^{-1}\right)$. Both the intensity and the envelope of the signal have been smoothed over $0.625 \mathrm{~ms}$, which corresponds to the time of 5 wave periods at the frequency of $80 \mathrm{kHz}$.

where $I$ is the acoustic intensity, or transport probability.

In a $3 \mathrm{D}$ infinite medium, $I(\mathbf{s}, \mathbf{r}, t)$ can be approximated by the solution of the diffusion equation:

$$
I^{i n f}(\mathbf{s}, \mathbf{r}, t)=\left(\frac{1}{4 \pi \mathcal{D} u}\right)^{\frac{3}{2}} \exp \left(\frac{-\|\mathbf{s}-\mathbf{r}\|^{2}}{4 \mathcal{D} t}\right) \exp (-\xi t),
$$

where $\|\mathbf{s}-\mathbf{r}\|$ measures the distance between $\mathbf{s}$ and $\mathbf{r}$. This approximation holds well provided that this distance is larger than the scattering mean free path $\ell^{\star}$. Given the experimental configuration, the propagation medium is simplified into a semi-infinite $35 \mathrm{~cm}$-thick concrete slab (Fig. 1c). We evaluate $I(\mathbf{s}, \mathbf{r}, u)$ taking into account the reflections at inner and outer surfaces of the tunnel by summing up the solutions $I^{\text {inf }}$ after the mirror principle. 
According to a preliminary calculation of sensitivity kernels, decorrelations observed from the sensor array are sensitive to microstructure changes over a $3.5 \mathrm{~m} \times 3.5 \mathrm{~m}$ area around the array (Fig. 1c). Therefore, for numerical computation the studied volume is $3.5 \mathrm{~m} \times 3.5 \mathrm{~m} \times 35 \mathrm{~cm}$ capturing the sensor network, and then meshed into 12500 cubic cells of size of $d \mathrm{v}=7 \times 7 \times 7 \mathrm{~cm}^{3}$. This spatial resolution of meshing is selected for being of the order of the transport mean free path $\left(\ell^{\star}\right)$.

With this propagation medium discretized, Eq. 1 can be represented as a matrix equation $\mathbf{d}=\mathbf{G m}^{\prime}$. The data vector $\mathbf{d}$ is filled with DC values while the distribution of microstructure changes is described by $\mathbf{m}^{\prime}$, a vector containing the value of $\sigma$ in every mesh cell. Matrix $\mathbf{G}$ relates linearly these two vectors, and its components are calculated using sensitivity kernels (Eq.1).

Deriving a model of $\mathbf{m}^{\prime}$, which estimates $\sigma$ in all 12500 mesh cells, from 1200 experimental DC values is an under-determined inverse problem. This is tackled by applying the method for discrete linear problem developed by Tarantola and Valette ${ }^{20}$ :

$$
\mathbf{m}=\mathbf{m}_{\mathbf{0}}+\mathbf{C}_{\mathbf{m}} \mathbf{G}^{\dagger}\left(\mathbf{G C}_{\mathbf{m}} \mathbf{G}^{\dagger}+\mathbf{C}_{\mathbf{d}}\right)^{-1}\left(\mathbf{d}-\mathbf{G m}_{\mathbf{0}}\right),
$$

where $\uparrow$ stands for matrix transpose. Assuming the components of $\mathbf{d}$ are independent, its covariance matrix $\mathbf{C}_{\mathbf{d}}$ is diagonal and defined based on an empirical model as $\mathbf{C}_{\mathbf{d}}=0.15 \mathbf{d} \mathbb{I}_{n}$, where $\mathbb{I}_{n}$ denotes the identity matrix of size of $n(n=1200$ here). The inversion is performed under the assumption that concrete is unchanged during the monitoring. Thus, no prior knowledge of the microstructure change is introduced in any of the cells. We only assume that the components of the inverted model $\mathbf{m}$ are independent. Therefore, the a priori model $\mathbf{m}_{\mathbf{0}}$ is filled with zeros and the covariance matrix of the model is defined as $\mathbf{C}_{\mathbf{m}}=s t d_{m}^{2} \mathbb{I}_{12500}$, where $s t d_{m}$ is the standard deviation of the model. $s t d_{m}$ is determined using the L-curve method so as to determine the best trade-off between the regularization of the model and the data fitting ${ }^{21}$. For more technical considerations about the inversion approach, the reader is invited to refer to previous works ${ }^{16}$.

\section{Experimental results}

The inverted model is represented in Fig. 3 which shows the distribution of $\sigma$ in the X-Y plane at three different depths: $3.5 \mathrm{~cm}, 17.5 \mathrm{~cm} \& 31.5 \mathrm{~cm}$. All 3 cracks can be distinguished clearly, and in particular the larger one in the center of the inspection zone $(\mathrm{x} \approx 2 \mathrm{~m})$. Their locations are in good agreement with observations made onsite at the outer surface (Fig. 1c), within a spatial uncertainty that corresponds to the

Prepared using sagej.cls 
scattering mean free path $\ell^{\star}$. Images reveal that cracks are fully penetrating through the thickness, which is consistent with the air leakage felt when passing hands above the cracks location during pressurization.
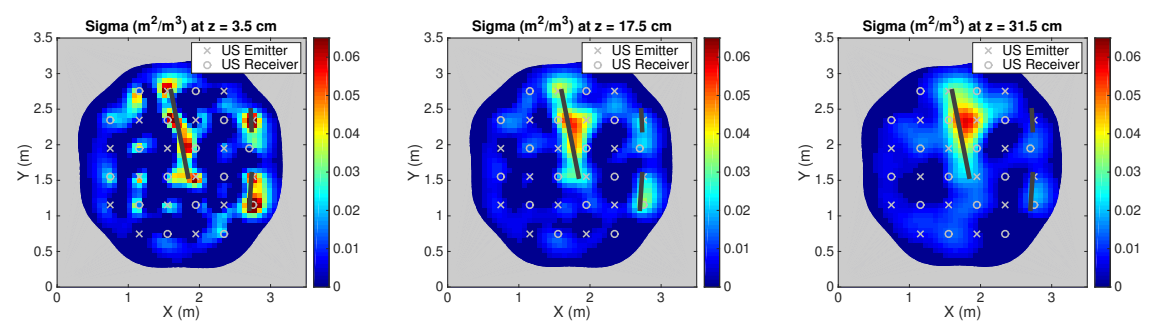

Figure 3. (Color online) Imaging of microstructure change occurring during a slight pressure release: the distribution of $\sigma$ in the $X-Y$ plane is mapped using color scale at three depths $(z=3.5 \mathrm{~cm}, 17.5 \mathrm{~cm} \& 31.5 \mathrm{~cm})$. The locations of cracks are indicated using black lines.

Note that a data exclusion criterion was introduced based on the resolution index of the inverted model ${ }^{16}$. A resolution index reported by Vergely et al. ${ }^{22}$ is calculated to evaluate the quality of the inverted model: the more this index is close to one, the 2 er the inverted model corresponds to the expected one ${ }^{23}$. A resolution index over one indicates that the inverted model might contain contribution from the error in experimental observations, while a resolution index close to zero indicates that the inverted model is dominated by the a priori model instead of the experimental observation, for instance due to a lack of sensitivity. Therefore, elements with a resolution index less than 0.25 are excluded, and the corresponding area in Fig. 3 is covered by a gray mask to avoid misinterpretations. In Fig. 4 the resolution index of the inverted model are illustrated accordingly at the same depths $(\mathrm{z}=3.5 \mathrm{~cm}, 17.5 \mathrm{~cm}$, and $31.5 \mathrm{~cm})$.
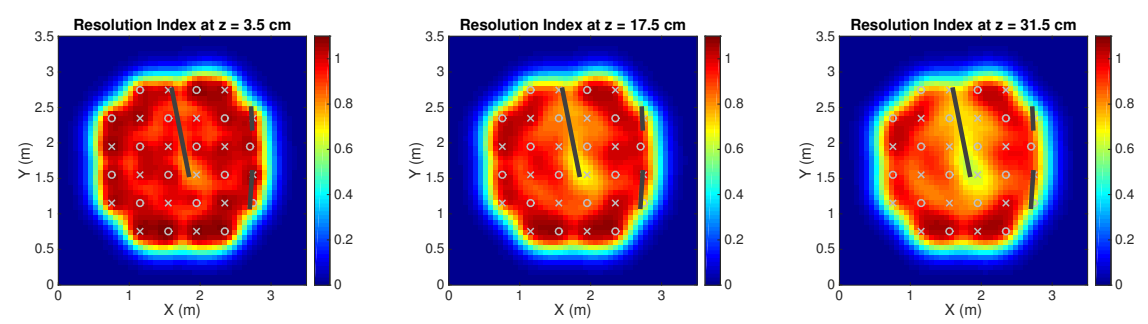

Figure 4. (Color online) The resolution index of the inverted model (Fig. 3) mapped using color scale at three depths $(z=3.5 \mathrm{~cm}, 17.5 \mathrm{~cm} \& 31.5 \mathrm{~cm})$. The locations of cracks are indicated using black lines. 
In the image, the three major signals correspond to three known macro-cracks, whose positions are confirmed through visual examin (10) from the surface of the concrete. And the elsewhere low signals indicate the absent of observable macroscopic cracks. This demonstrates that Locadiff is a reliable method for practical on-site applications on real industrial infrastructures, provided structural changes, even very small ones, during monitoring. Of particular interest is the resolution of the cracks at depth, which can hardly be achieved by other on-site non-destructive techniques. Also, the localisation of the crack end at the center of the image is of interest, since it coincides well with surface observations and demonstrates that the central main crack does not continue in the bottom direction $(\mathrm{Y}<1.5 \mathrm{~m})$. On the final image, we can observe low amplitude signals (for instance at $X=1.2 \mathrm{~m}, \mathrm{Y}=2.5 \mathrm{~m}$ ), which can be either uncertainty fluctuations related to the methodology, or to internal minor damage subject to future developments. Such observations fixe the level at which signal can no longer be interpreted yet.

\section{Conclusions \& Discussions}

In this study, we used diffuse ultrasound processed with the Locadiff imaging technique on an aeronautical wind tunnel to locate and characterize three pre-existing cracks in the structure. The major finding of the work is that the high sensitivity of Locadiff allows the characterization of cracks with very limited sollicitations. Indeed, a pressure change lower than $1 \mathrm{kPa}$ was enough to induce structural changes (micro-deformation) at the cracks such that they become visible to diffuse ultrasounds. Such micro-metric changes can be either crack opening, or longitudinal displacement along the crack surface. Results also demonstrate the ability of the method to image pre-existing cracks in concrete, in terms of robustness and feasibility: 1) the inversion procedure does not need excessive optimization: the propagation medium is simplified (infinite slab) and the inversion parameters are approximations of the transport parameters of diffuse ultrasounds; and 2) experiments are fully non-destructive and can be performed on site.

In addition, since structural changes due to crack opening/closing can also be triggered by other sollicitations, we believe that this technique can be largely applied. For example, similar experiments could be performed using small temperature changes $\left(<1^{\circ} \mathrm{C}\right)$ within the material for which thermal expansion will induce local deformations at the cracks. In its current state this technique requires the two surfaces of the cracks to be in a state of imperfect contact. However, authors are confident that this is not a very restricting condition, especially in pre-stressed concrete structures.

Prepared using sagej.cls 


\section{Acknowledgements}

This work was partially funded by the ANR (PIA) ENDE, the VOR program from the Univ. Grenoble Alpes, and is part of the Labex OSUG@2020. The authors would like to thank B. Valette and T. Planès for enlightening discussions.

\section{References}

1. Planès $\mathrm{T}$ and Larose E. A review of ultrasonic Coda Wave Interferometry in concrete. Cem Concr Res 2013; 53: 248-255. DOI:10.1016/j.cemconres.2013.07.009. URL http: //linkinghub.elsevier.com/retrieve/pii/s0008884613001701.

2. Aki K. Analysis of the seismic coda of local earthquakes as scattered waves. $J$ Geophys Res 1969; 74(2): 615-631. DOI:10.1029/JB074i002p00615. URL http: // doi.wiley.com/10.1029/JB074i002p00615http: / / onlinelibrary . wiley.com/doi/10.1029/JB074i002p00615/abstract.

3. Snieder R. Coda wave interferometry and the equilibration of energy in elastic media. Phys Rev E 2002; 66(4): 046615. DOI:10.1103/PhysRevE.66.046615. URL http: //link.aps.org/doi/10.1103/PhysRevE.66.046615.

4. Larose E, de Rosny J, Margerin L et al. Observation of multiple scattering of $\mathrm{kHz}$ vibrations in a concrete structure and application to monitoring weak changes. Phys Rev E 2006; 73(1): 016609. DOI:10.1103/PhysRevE.73.016609. URL http://link.aps.org/ doi/10.1103/PhysReve.73.016609.

5. Grêt A, Snieder R and Scales J. Time-lapse monitoring of rock properties with coda wave interferometry. J Geophys Res Solid Earth 2006; 111(B3): n/a-n/a. DOI:10.1029/ 2004JB003354. URL http: / / doi.wiley. com/10 .1029/2004 JB003354.

6. Larose E and Hall S. Monitoring stress related velocity variation in concrete with a 2105 relative resolution using diffuse ultrasound. J Acoust Soc Am 2009; 125(4): 18531856. DOI:10.1121/1.3079771. URL http://scitation.aip.org/content/ asa/journal/jasa/125/4/10.1121/1.3079771.

7. Payan C, Garnier V, Moysan J et al. Determination of third order elastic constants in a complex solid applying coda wave interferometry. Appl Phys Lett 2009; 94(1): 011904. DOI:10.1063/1.3064129. URL http://link.aip.org/link/?APL/94/ $011904 / 1$ http://scitation.aip.org/content/aip/journal/apl/94/ $1 / 10.1063 / 1.3064129$.

8. Larose E, Roux P, Campillo M et al. Fluctuations of correlations and Green's function reconstruction: Role of scattering. J Appl Phys 2008; 103(11): 114907. DOI:10.1063/ 1.2939267. URL http://scitation.aip.org/content/aip/journal/jap/ 103/11/10.1063/1.2939267.0805.0195.

Prepared using sagej.cls 
9. Schurr DP, Kim JY, Sabra KG et al. Damage detection in concrete using coda wave interferometry. NDT E Int 2011; 44(8): 728-735. DOI:10.1016/j.ndteint.2011.07. 009. URL http://dx.doi.org/10.1016/j.ndteint.2011.07.009http: //linkinghub.elsevier.com/retrieve/pii/s0963869511000983.

10. Zhang Y, Abraham O, Grondin F et al. Study of stress-induced velocity variation in concrete under direct tensile force and monitoring of the damage level by using thermally-compensated Coda Wave Interferometry. Ultrasonics 2012; 52(8): 10381045. DOI:10.1016/j.ultras.2012.08.011. URL http://dx.doi.org/10.1016/ j.ultras.2012.08.011http://linkinghub.elsevier.com/retrieve/ pii/s0041624X12001643.

11. Hilloulin B, Zhang Y, Abraham $\mathrm{O}$ et al. Small crack detection in cementitious materials using nonlinear coda wave modulation. NDT \& E Int 2014; 68: 98-104. DOI:10.1016/j.ndteint.2014.08.010. URL http://dx.doi.org/10.1016/j. ndteint.2014.08.010http://linkinghub.elsevier.com/retrieve/ pii/s0963869514001133.

12. Larose E, Planes $\mathrm{T}$, Rossetto $\mathrm{V}$ et al. Locating a small change in a multiple scattering environment. Appl Phys Lett 2010; 96(20): 204101. DOI:10.1063/1. 3431269. URL http://scitation.aip.org/content/aip/journal/apl/ 96/20/10.1063/1.3431269.

13. Rossetto V, Margerin L, Planes $\mathrm{T}$ et al. Locating a weak change using diffuse waves: Theoretical approach and inversion procedure. J Appl Phys 2011; 109(3): 034903. DOI:10. 1063/1.3544503. URL http://scitation.aip.org/content/aip/journal/ jap/109/3/10.1063/1.3544503.

14. Planès T, Larose E, Margerin L et al. Decorrelation and phase-shift of coda waves induced by local changes: multiple scattering approach and numerical validation. WAVE RANDOM COMPLEX 2014; 24(2): 99-125. DOI:10.1080/17455030.2014.880821. URL http: / / www.tandfonline.com/doi/abs/10.1080/17455030.2014. $880821 \#$.Vo4zffnhBaQ.

15. Larose E, Obermann A, Digulescu A et al. Locating and characterizing a crack in concrete with diffuse ultrasound: A four-point bending test. J Acoust Soc Am 2015; 138(1): 232-241. DOI:10.1121/1.4922330. URL http: / / scitation.aip.org/content/ asa/journal/jasa/138/1/10.1121/1.4922330.

16. Zhang Y, Planès T, Larose E et al. Diffuse ultrasound monitoring of stress and damage development on a 15-ton concrete beam. J Acoust Soc Am 2016; 139(4): 16911701. DOI:10.1121/1.4945097. URL http://scitation.aip.org/content/ asa/journal/jasa/139/4/10.1121/1.4945097.

Prepared using sagej.cls 
17. Xie F, Moreau L, Zhang Y et al. A Bayesian approach for high resolution imaging of small changes in multiple scattering media. Ultrasonics 2016; 64: 106-114. DOI:10.1016/j.ultras.2015.08.005. URL http://dx.doi.org/10.1016/j. ultras.2015.08.005http://linkinghub.elsevier.com/retrieve/ pii/s0041624X15002152.

18. Lobkis OI and Weaver RL. Coda-wave interferometry in finite solids: Recovery of $p$-to- $s$ conversion rates in an elastodynamic billiard. Phys Rev Lett 2003; 90(25): 254302. DOI: 10.1103/PhysRevLett.90.254302. URL http://link.aps.org/doi/10.1103/ PhysRevLett. 90.254302.

19. Sens-Schönfelder C and Wegler U. Passive image interferemetry and seasonal variations of seismic velocities at Merapi Volcano, Indonesia. Geophys Res Lett 2006; 33(21): 1-5. DOI:10.1029/2006GL027797. URL http://doi.wiley.com/10.1029/ 2006 GL027797.

20. Tarantola A and Valette B. Generalized nonlinear inverse problems solved using the least squares criterion. Rev Geophys 1982; 20(2): 219. DOI:10.1029/RG020i002p00219. URL http://dx.doi.org/10.1029/RG020i002p00219http://doi.wiley. com/10.1029/RG020i002p00219.

21. Hansen PC. Analysis of Discrete Ill-Posed Problems by Means of the L-Curve. SIAM Rev 1992; 34(4): 561-580. DOI:10.1137/1034115.

22. Vergely, J-L and Valette, B. and Lallement, R. and Raimond, S. Spatial distribution of interstellar dust in the Sun's vicinity: comparison with neutral sodium-bearing gas. Astron Astrophys 2010; 518: A31. DOI:10.1051/0004-6361/200913962. URL https: //hal.archives-ouvertes.fr/hal-00476261.

23. Obermann, A and Froment, $\mathrm{B}$ and Campillo, $\mathrm{M}$ and Larose, $\mathrm{E}$ and Planès, $\mathrm{T}$ and Valette, B and Chen, J H and Liu, Q Y Seismic noise correlations to image structural and mechanical changes associated with the Mw 7.9 2008 Wenchuan earthquake. J Geophys Res Solid Earth 2014; 119(4): 3155-3168. DOI:10.1002/2013JB010932. URL http: //dx.doi.org/10.1002/2013JB010932.

Prepared using sagej.cls 